Published in final edited form as:

J Cardiovasc Transl Res. 2017 June ; 10(3): 233-244. doi:10.1007/s12265-017-9756-y.

\title{
Precision Medicine for Heart Failure with Preserved Ejection Fraction: An Overview
}

\author{
Sanjiv J. Shah, MD \\ Division of Cardiology, Department of Medicine, Northwestern University Feinberg School of \\ Medicine, Chicago, Illinois
}

\begin{abstract}
There are few proven therapies for heart failure with preserved ejection fraction (HFpEF). The lack of therapies, along with increased recognition of the disorder and its underlying pathophysiology, has led to the acknowledgement that HFpEF is heterogeneous and is not likely to respond to a one-size-fits-all approach. Thus, HFpEF is a prime candidate to benefit from a precision medicine approach. For this reason, we have assembled a compendium of papers on the topic of precision medicine in HFpEF in the Journal of Cardiovascular Translational Research. These papers cover a variety of topics relevant to precision medicine in HFpEF, including automated identification of HFpEF patients; machine learning, novel molecular approaches, genomics, and deep phenotyping of HFpEF; and clinical trial designs that can be used to advance precision medicine in HFpEF. In this introductory article, we provide an overview of precision medicine in HFpEF with the hope that the work described here and in the other papers in this special theme issue will stimulate investigators and clinicians to advance a more targeted approach to HFpEF classification and treatment.
\end{abstract}

\section{Keywords}

heart failure with preserved ejection fraction; precision medicine; machine learning; cluster analysis; treatment

\section{Introduction}

The field of precision medicine [1] promises to introduce greater personalization of treatments for heterogeneous clinical syndromes such as heart failure (HF). Although there is already rudimentary classification of HF that is used to target treatment in HF (e.g., HF with reduced ejection fraction [HFrEF] vs. HF with preserved ejection fraction [HFpEF]), a

\footnotetext{
Corresponding author: Sanjiv J. Shah, MD, Professor of Medicine, Division of Cardiology, Department of Medicine, Northwestern University Feinberg School of Medicine, Phone: 312-695-0993, Fax: 312-253-4470, sanjiv.shah@northwestern.edu. COMPLIANCE WITH ETHICAL STANDARDS

Conflicts of interest: Dr. Shah has received research grants from Actelion, AstraZeneca, Corvia, and Novartis; and consulting fees from Actelion, Amgen, AstraZeneca, Bayer, Boehringer-Ingelheim, Cardiora, Eisai, Ironwood, Merck, MyoKardia, Novartis, Pfizer, Sanofi, and United Therapeutics.

Ethical approval: This review article does not contain any primary data from studies with human participants or animals performed by the author.

Informed consent: Not applicable (this paper is a review article with no primary research involved).
} 
true precision medicine approach to HF is currently in its infancy, and treatment of $\mathrm{HFpEF}$ and HFrEF patients is still based on a "one-size-fits-all" approach [2]. HFpEF is particularly challenging because it has proven to be highly heterogeneous and resistant to conventional therapies that have worked for HFrEF. Thus, HFpEF may be an ideal candidate for a precision medicine approach.

Although it seems clear that a more personalized, precision medicine approach to the treatment of the individual HF patient would be beneficial, the contemporary definitions of "precision medicine" are somewhat problematic. The National Institutes of Health (NIH) defines precision medicine as "... an emerging approach for disease treatment and prevention that takes into account individual variability in genes, environment, and lifestyle for each person" [3]. While this statement is accurate, the current focus of precision medicine seems to be on the collection of -omics type "big data" (particularly genetic data) that will somehow result in the development of personalized therapeutics for patients [1]. Missing from these definitions of precision medicine is the central tenet that the reason to obtain "big data" is so that we can resolve heterogeneity by identifying patterns within the data that enable the identification of subtypes of patients within a particular disease or clinical syndrome or predicting response to therapy. The key to identification of these patterns is machine learning [4].

Machine learning, which was developed within the fields of statistics and computer science over the last several decades, is focused on using computer algorithms to learn from data. In most instances, the ultimate goal of machine learning is to learn from existing data in order to make sense of new data. Machine learning is thus likely to be an essential and increasingly frequent part of the future of precision medicine.

In this special issue of the Journal of Cardiovascular Translational Research (JCTR), we have put together a compendium of original research and review articles on the topic of precision medicine in HFpEF that incorporates advances in automated patient identification, big data, and machine learning analytics [5-10]. These papers cover a variety of topics relevant to precision medicine, including text mining and natural language processing to identify HFpEF patients [11]; tensor factorization, a promising machine learning technique that can be applied to the field of HFpEF [10]; molecular approaches to precision medicine in HFpEF (induced pluripotent stem cell-derived cardiomyocytes and microRNAs) [9]; genome-wide characterization of molecular profiles of HFpEF [7]; deep phenotyping of arterial hemodynamics in HFpEF [5,6]; phenomapping of hypertension to identify the myocardial substrate for HFpEF [8]; and finally innovative clinical trial designs that can be used to advance precision medicine in HFpEF [12].

In this introductory paper, we provide an overview of precision medicine in $\mathrm{HFpEF}$ in order to set the stage for this special issue of JCTR. Here we discuss the challenge of the HFpEF syndrome; provide an overview of machine learning as it applies to HFpEF; discuss 3 archetypes of HFpEF; briefly outline strategies for targeted treatment of HFpEF; and conclude with future directions for precision medicine in HFpEF. 


\section{The Challenge of HFpEF}

Despite advances in our understanding of the diagnosis, pathophysiology, and treatment of HFpEF [13], it remains one of the most challenging clinical syndromes in cardiovascular medicine for several reasons. First, HFpEF prevalence is increasing with the increasing age of the population and the epidemics of obesity, hypertension, and diabetes [14]. For instance, based on extrapolation of HF hospitalization trends from the Get With the Guidelines Heart Failure Study (2005-2010), by the year 2020, 50\% of hospitalized HF patients in the United States will have an EF > 50\%, and 65\% of hospitalized HF patients will have an EF > 40\% [15]. Given the general lack of proven therapies for patients with $\mathrm{HF}$ and $\mathrm{EF}>40 \%$, this alarming trend constitutes a major public health problem. Second, HFpEF is associated with high morbidity. As shown in the TOPCAT trial participants, all of whom had chronic HFpEF, quality of life is very poor-worse than HFrEF and similar to that seen in end-stage renal disease patients on dialysis [16]. Furthermore, activity level is very low, similar to levels in moderate or greater chronic obstructive pulmonary disease [16]. Third, survival of $\mathrm{HFpEF}$ is poor, particularly after HF hospitalization. In one landmark HFpEF epidemiology study published in 2006, survival of both HFpEF and HFrEF was a dismal 35\% at 5 years after HF hospitalization [17]. While subsequent studies have demonstrated higher mortality in HFrEF compared to HFpEF (likely due to different settings in which patients are enrolled - epidemiology studies vs. registries vs. clinical trials), regardless of comparison to HFrEF, mortality rates in HFpEF are quite high. Fourth, one of the most difficult problems in $\mathrm{HFpEF}$ is the presence of numerous pathophysiologic abnormalities in the heart and other organs [18]. Unlike HFrEF, in which neurohormonal activation is a central theme that dominates the pathophysiology after the initial myocardial insult, HFpEF patients can have highly variable underlying pathophysiologies that contribute to the heterogeneity of the syndrome [2]. Finally, results from large-scale clinical trials in HFpEF have been disappointing for the most part. Although Phase 3 trials of spironolactone (TOPCAT) [19] and implantable pulmonary artery hemodynamic sensors (CHAMPION) [20] have demonstrated a reduction in HF hospitalization, several other trials (e.g., CHARM-Preserved [candesartan] [21], I-PRESERVE [irbesartan] [22], RELAX [sildenafil] [23], and NEAT [isosorbide mononitrate] [24]) showed no benefit.

Given all of these challenges in HFpEF, coupled with the heterogeneity of the HFpEF syndrome, we are in dire need of new approaches. Over the past 10-15 years, our understanding of HFpEF has evolved (Figure 1), which has helped us get closer to developing effective treatments for HFpEF patients [13,25-27]. We now understand that HFpEF is not simply a clinical syndrome consisting of a small LV, significant concentric LV hypertrophy, normal EF, and diastolic dysfunction with reduced LV diastolic compliance. HFpEF is a multi-organ system disease that involves not only the heart but also the lungs, skeletal muscle, kidneys, and adipose tissue [18]. Hypertensive remodeling; sedentary lifestyle with poor fitness; ventricular and vascular stiffening; obesity and metabolic stress; and aging, coupled with widespread endothelial dysfunction in several organs leads to a global loss in cardiac, skeletal, and peripheral reserve, which ultimately results in the HFpEF syndrome [27]. 
In order to grapple with the multi-system nature of $\mathrm{HFpEF}$ and the challenges described above, one solution is the development of dedicated HFpEF clinical and research programs, as discussed in detail elsewhere [28]. The development of these specialized HFpEF programs enables the comprehensive study and care of HFpEF patients. Furthermore, as these programs care for large numbers of HFpEF patients, they can successfully categorize and treat patients across the phenotypic spectrum. Precision medicine in HFpEF starts with improved classification of these patients into therapeutically homogeneous patient subtypes. Table 1 demonstrates various published methods of classifying HFpEF [29,30,2,31,32], most of which originated from centers that take care of a large number of HFpEF patients in a focused manner.

\section{Machine Learning to Advance Precision Medicine in HFpEF}

As mentioned above, machine learning methodologies are poised to help advance precision medicine in HFpEF [4]. Machine learning is not new, and it has already been applied successfully to several industries, but is only now beginning to be applied to healthcare. Machine learning is more likely to advance precision medicine compared to conventional statistical techniques because it is able to handle large amounts of data with complex interactions between variables. Machine learning can be broadly divided into supervised learning and unsupervised learning [4].

Supervised machine learning is based on a defined outcome (i.e., "outcomes" are labeled). At a basic level, the commonly used logistic regression and Cox regression analyses are forms of supervised machine learning. However, these types of regression analyses assume that variables are linearly associated with each other, and cannot simultaneously examine higher-order interactions among variables (e.g., phenotypes) within the dataset. Examples of supervised learning techniques include support vector machines and random forests. Unsupervised machine learning is based on intrinsic patterns in the data without prior knowledge of the outcome. Determining subtypes of HFpEF in a deeply phenotyped dataset is an example of unsupervised learning. Cluster analyses, such as hierarchical clustering, $\mathrm{K}-$ means clustering, and model-based clustering are examples of unsupervised learning analyses.

Deep learning is a branch of machine learning that is increasing in popularity due to its ability to process highly non-linear data that requires modeling of increasingly higher level of abstractions across multiple processing layers [33]. Deep learning is currently most often used to perform supervised learning tasks but can also be used for unsupervised learning. Examples of uses of deep learning in medicine include automated processing and diagnosis of medical images (e.g., skin photographs for dermatologic diagnoses [34], and retinal photographs for diagnosing diabetic retinopathy [35]), and for making clinical predictions from large amounts of data from the electronic health record, a process that has been termed "deep patient" [36].

As mentioned above, the current focus on precision medicine is on -omics type data, particularly genomic data. However, the data that is used for machine learning can be of any type (e.g., -omics of any kind; environmental data; lifestyle data such as accelerometry; 
electronic health record data). Indeed for many clinical syndromes, such as HFpEF, the genomic-centric approach may not be ideal. Besides being a complex clinical syndrome with multiple etiologies and pathophysiologies (as outlined above), HFpEF has a limited genetic/ heritable component in most instances. Furthermore, in HFpEF accessing the diseased tissue (particularly myocardial tissue) is not readily available; thus large-scale gene expression analyses and tissue characterization of the heart and other organs is typically not possible. Thus, for many clinical syndromes such as HFpEF, we must leverage non-genetic data, coupled with machine learning, in order to resolve the heterogeneity of the HFpEF syndrome, which will hopefully allow for more targeted therapeutics.

With improvements in phenotyping techniques, including imaging, proteomics, and metabolomics, we can take advantage of modern, "big data" analytics. We have shown in HFpEF that the combination of deep echocardiographic phenotyping with unsupervised model-based clustering-based machine learning (i.e., "phenomapping" [Figure 2]) resulted in the detection of 3 mutually exclusive subcategories of HFpEF that differed greatly in their clinical characteristics, pathophysiology, and outcomes (Figure 3) [31]. Pheno-group \#1 patients had the lowest BNP, were the youngest, had the least abnormalities in cardiac structure and function, were obese, and had the best outcomes. Pheno-group \#2 patients had higher BNP levels, had the worse LV relaxation (lowest e' velocity), and had the highest prevalence of diabetes and obesity. Pheno-group \#3 patients had the highest prevalence of electrocardiographic abnormalities, RV dysfunction, pulmonary hypertension, and renal dysfunction, and these patients had the worst outcomes.

Although much more work needs to be done to validate a phenomapping approach to $\mathrm{HFpEF}$, moving towards such an approach in HFpEF seems attractive. One could imagine that at some point in the future, once the diagnosis of HFpEF is made, patients would undergo physical examination, phlebotomy, electrocardiography, and echocardiography, with all of the resultant quantitative data entered into an algorithm and the pheno-group assigned, after which patients would be guided towards specific therapies and clinical trials. However, this approach would require systematic evaluation of the echocardiogram by a physician or technician with extensive expertise in quantitative echocardiographic analysis, as was done in the original HFpEF phenomapping study. Such an approach does not seem feasible for widespread adoption of phenomapping in HFpEF. Therefore, a more automated way to analyze echocardiograms from HFpEF patients is necessary.

Recently, as mentioned above, deep learning has been applied to medical images. Besides the published examples of deep learning for automated analysis of skin photographs and retinal photographs, there are currently ongoing studies of deep learning for radiology images. These techniques could also be applied to echocardiograms. Thus, in the future, deep learning and related techniques could be applied to echocardiograms to derive echocardiographic features (phenotypes), and these could be then combined with other data to more efficiently conduct the phenomapping of HFpEF patients and assign them to phenogroups in order to determine the best treatment pathway and clinical trials (Figure 4). 


\section{The 3 archetypes of HFpEF}

As shown in Figure 1, the understanding of HFpEF pathophysiology has evolved over the last 10-15 years. The emerging model of HFpEF implicates comorbidity-induced systemic inflammation in the pathogenesis of endothelial dysfunction, myocardial fibrosis, and cardiomyocyte stiffening (via abnormalities in titin, the large molecular spring within cardiomyocytes).[37] Several investigators have shown that cyclic GMP-protein kinase G (cGMP-PKG) molecular signaling system to be very important in the pathogenesis of HFpEF, not only in the heart, but also in other affected organs such as the lung, adipose tissue, skeletal muscle, and the kidney [3,38-42]. However, even if deficiency of the cGMPPKG signaling system is a dominant underlying cause of HFpEF, it is likely not the only one, and differential abnormalities of cGMP-PKG in various organs could account for the heterogeneity of the HFpEF syndrome. Thus, improved understanding of the various types of HFpEF is essential. In this section, we discuss the potential mechanisms underlying the 3 HFpEF archetypes identified in the HFpEF phenomapping study (Figure 3): the natriuretic peptide (NP) deficiency syndrome phenotype; the obesity-cardiometabolic phenotype; and the RV failure, cardiorenal phenotype.

\section{HFpEF pheno-group \#1: Natriuretic peptide deficiency syndrome}

Pheno-group \#1 is characterized by low BNP. It is now clear, based on several studies, that $\mathrm{BNP}$ is below the range typically considered diagnostic of $\mathrm{HF}$ (e.g., BNP $<100 \mathrm{pg} / \mathrm{ml}$ ) in approximately $30 \%$ of patients with HFpEF [43]. There are several reasons why these patients may have a low BNP, but it is likely that an "NP deficiency syndrome" exists in many of these patients and could be a major underlying cause their HFpEF syndrome. It is well known that obesity is a major cause of low BNP levels due to increased BNP clearance and reduced BNP production [44-46]. However, it is less well recognized that NPs stimulate browning of fat, thereby increasing the amount of healthy adipose tissue [3,47]. Thus, a low NP level perpetuates itself by increasing the amount of metabolically deranged adipose tissue (which in turn leads to reduced NP levels for the reasons stated above). Furthermore, inability to generate NPs results in sodium retention and elevated blood pressure, both of which are major contributors to HFpEF. Obesity is not the only cause of the NP deficiency syndrome. Several other factors, including genetic polymorphisms in the NPPA and NPPB genes [48], African ancestry [49], insulin resistance [45], increased androgens [50], and possibly even increased activity of neprilysin could all result in low levels of NPs, which contribute to and worsen the HFpEF syndrome, thereby potentially leading to a type of HFpEF consistent with pheno-group \#1.

There also appears to be a specific obesity HFpEF phenotype that is unique and different than other forms of HFpEF, and may be present in some pheno-group \#1 patients. In a recent study by Obokata et al., obese HFpEF (BMI > $35 \mathrm{~kg} / \mathrm{m}^{2}$ ), non-obese HFpEF, and controls underwent a detailed evaluation with plasma volume measurement, invasive hemodynamic testing at rest and during exercise, and echocardiography [51]. These investigators found that compared to non-obese HFpEF, obese HFpEF patients had lower BNP levels, greater plasma volume, increased total heart size, and higher right heart pressures compared to left heart pressures at rest and during exercise. In addition, obese HFpEF patients had increased 
epicardial fat and greater interventricular septal flattening resulting in greater eccentricity of the LV. These findings suggest that the increased plasma volume and greater central adiposity (including epicardial fat) create ventricular interdependence and pericardial restraint, which gives rise to the HFpEF syndrome.

In summary, pheno-group \#1 may represent an NP deficiency syndrome phenotype of HFpEF. The NP deficiency results in the HFpEF syndrome and perpetuates it, and the lack of NP may require specialized treatment. Unfortunately, because these pheno-group \#1 patients have low NP levels and elevated body mass index (BMI), the diagnosis is often missed despite the frequent finding of very high LV filling pressures in these patients. Furthermore, these patients are often excluded from HFpEF clinical trials because their BMI is higher than the cut-off for inclusion into trials or NP levels are too low. Thus, pheno-group \#1 patients represent a major unmet need in HFpEF in terms of both diagnosis and treatment. The exclusion of these types of patients can have major consequences. For example, in TOCPAT, the patients with the lowest NP levels were most likely to benefit from spironolactone [52]. In PARAGON, the ongoing phase 3 trial of sacubitril/valsartan (an angiotensin receptor-neprilysin inhibitor), all patients are required to have an elevated $\mathrm{N}$ terminal pro-BNP level, despite the fact that NP-deficient HFpEF patients could be the ones that benefit the most from inhibiting neprilysin, which breaks down BNP.

\section{HFpEF pheno-group \#2: Obesity-cardiometabolic phenotype}

Cardiometabolic disease, which encompasses systemic hypertension, obesity, insulin resistance (i.e., type 2 diabetes), and the metabolic syndrome, is increasingly common in the population and a major cause of HFpEF. In fact, one could view the HFpEF syndrome as "cardiometabolic disease to the extreme". Pheno-group \#2 had the highest prevalence of diabetes in the HFpEF phenomapping study, and appeared to have more intrinsic myocardial dysfunction than pheno-group \#1, with significantly reduced tissue Doppler e' velocities, which is indicative of severely impaired LV relaxation. We have previously shown that early in response to hypertension, cardiomyocytes develop T-tubule disruption, which correlates with abnormalities in cardiac mechanics identified by tissue Doppler and speckle-tracking strain analysis [53]. T-tubules are the passageways by which calcium shuttles around the cardiomyocytes, and are therefore essential to efficient cardiomyocyte calcium release and uptake, which underlies contraction and relaxation, respectively [54]. We hypothesize that pheno-group \#2 patients have T-tubule disruption, which results in abnormal calcium handling within cardiomyocytes, which, in turn, leads to abnormal cardiac mechanics, particularly reduced e' velocity and longitudinal strain. Coupled with cardiac microvascular dysfunction, T-tubule disruption in response to stressors like hypertension could lead to significantly impaired subednocardial dysfunction, which results in abnormal longitudinal myocardial mechanics, thereby leading to the HFpEF syndrome. Central aortic stiffening also likely exacerbates HFpEF in pheno-group \#2. As cardiomyocyte dysfunction worsens, peak systolic stress of the LV occurs later in systole. Meanwhile, as the aorta stiffens, the reflected aortic pulse wave grows in magnitude and comes earlier in systole. These abnormalities likely combine to increase T-tubule disruption, thereby worsening cardiac mechanics and propagating the HFpEF syndrome [55]. 
In summary, pheno-group \#2 patients have an obesity-cardiometabolic phenotype, which may lead to abnormal ventricular-arterial coupling and abnormal cardiac mechanics. Furthermore, the majority of pheno-group \#2 patients have a very high BMI $\left(>35 \mathrm{~kg} / \mathrm{m}^{2}\right)$ and thus may also suffer from the obesity HFpEF phenotype described above. Based on the aforementioned pathophysiology, pheno-group \#2 patients may respond best to drugs that target microvascular dysfunction or the cardiometabolic phenotype (e.g., SGLT-2 inhibitors [56]) or even medical or surgical weight loss strategies. Theoretically, controlling blood pressure would also help these patients; however, thus far reducing blood pressure has not led to major improvements in outcomes in HFpEF patients based on trials of angiotensin receptor blockers and angiotensin converting enzyme inhibitors. In these HFpEF trials, blood pressure lowering occurred to a similar magnitude to hypertension trials, without the improvement in outcomes seen in the hypertension trials [25].

\section{HFpEF pheno-group \#3: Right ventricular failure, cardiorenal phenotype}

As shown in Figure 2, HFpEF patients in pheno-group \#3 had the worst outcomes. These patients had an RV failure, cardiorenal phenotype. In HFpEF, chronic kidney disease (CKD) is associated with worse RV dysfunction and higher pulmonary artery (PA) pressures,[57] the causes of which are likely multifactorial and bidirectional. First, CKD causes volume overload, increasing left atrial (LA) pressure and stress on the LA and pulmonary veins. These abnormalities, in turn, increase PA pressure over time, thereby leading to RV dysfunction. RV dysfunction can also lead to renal venous congestion, which would worsen renal function.

An additional intriguing pathophysiologic pathway may also be at play in the pathogenesis of pheno-group \#3. Worsening renal function could lead to reduced clearance of phosphate, thereby leading to increased levels of the phosphaturic hormone FGF23 and secondary hyperparathyroidism, which may lead to increased levels of calcium and ectopic calcification of the pulmonary vessels. Indeed in a canine study of CKD (via nephrectomy), PA pressures were higher and pulmonary vascular calcification was present, along with elevated levels of parathyroid hormone [58]. In the same study, pre-treating the dogs with parathyroidectomy prior to inducing CKD prevented the development of pulmonary hypertension. Thus, in pheno-group \#3, ectopic calcification of the pulmonary vessels may occur in response to CKD, which could be an additional contributor to increased RV afterload with worsening RV dysfunction and right-sided HF.

In summary, RV dysfunction and renal dysfunction combine in a bidirectional pattern in HFpEF pheno-group \#3, often leading to a downward spiral that leads to worse cardiovascular outcomes and death. Treatment of pheno-group \#3 is quite challenging. These patients tend to have end-stage disease and therefore may not respond to treatments. In addition, although there have been trials of pulmonary vasodilators in HF (including $\mathrm{HFpEF}$ ), right-sided heart failure is essentially ignored in HF clinical trials despite being a major unmet need. 


\section{Management of HFpEF in the Era of Precision Medicine}

Management of HFpEF in the current era of precision medicine depends on accurate diagnosis and classification of these patients. Typically, once HFpEF is diagnosed, we must first exclude those patients with alternate causes of the HFpEF syndrome (e.g., constrictive pericarditis, high-output $\mathrm{HF}$, severe valvular disease, HFrEF with recovered $\mathrm{EF}$, and infiltrative and genetic cardiomyopathies), so that we can get to more common causes of the HFpEF syndrome. However, even after sorting HFpEF patients and focusing on common forms of $\mathrm{HFpEF}$, treatment is challenging, and detailed treatment recommendations are beyond the scope of this review. Nevertheless, we have recently developed a phenotypespecific treatment paradigm that provides a "HFpEF treatment matrix" (Figure 5) that provides a starting point towards more personalized treatment for HFpEF [25]. In the $\mathrm{HFpEF}$ treatment matrix, phenotype-specific treatment of HFpEF is based on a combination of the predisposing risk factors and the type of clinical presentation. Additional published reviews have also outlined etiology-specific treatments that can be helpful in creating a more personalized approach to the management of HFpEF [59].

\section{The Future of Precision Medicine in HFpEF}

What is required for the advancement of precision medicine in HFpEF? First, we need deeply phenotyped and curated data from large cohorts of patients with HFpEF, and these cohorts would ideally be phenotyped in multiple orthogonal domains. The development of these types of HFpEF cohorts will require dedicated HFpEF clinical and research programs. In addition, large-scale identification of HFpEF patients for precision medicine studies will require novel ways of interrogating the electronic health record with techniques such as natural language processing. Once HFpEF patients are identified, they can be further categorized using deep phenotyping registry studies and then directed towards the HFpEF clinical trial that matches best with their underlying pathophysiologic features. Figure 6 displays this type of framework with HFpEF registries and clinical trials that are currently being conducted in the Northwestern University HFpEF Program.

Although machine learning is likely to assist with the advancement of precision medicine in HFpEF, only properly executed machine learning studies will be useful. As mentioned above, at the heart of machine learning is training and validation. Thus, at the onset of study design, investigators should also develop an external validation cohort. The machine learning algorithm(s) must first be trained and tuned (and internally validated) on 1 dataset; once the machine learning algorithm has been created the next step is validating its utility and efficacy in a separate, external dataset. Furthermore, the machine learning approach should be shown to be superior to more conventional and simpler statistical techniques. Finally, once various subtypes of HFpEF are identified by machine learning, further studies should be conducted to determine the pathophysiologic and molecular differences among the HFpEF subtypes, and whether these subtypes respond differently to various therapies. 


\section{Conclusions}

HFpEF is a major public health problem that is associated with high morbidity and mortality and few proven treatment options. Given the heterogeneity of the HFpEF syndrome, it is an ideal disorder to benefit from precision medicine. High-resolution phenotyping and analysis of genomics, proteomics, metabolomics, and exposures, coupled with machine learning and patient-specific cellular models holds great promise and is the focus of this issue of JCTR. While there is tremendous excitement for the role of precision medicine in HFpEF, we must remember to use the highest standards for conducting and interpreting precision medicine studies, and a healthy amount of skepticism for newer techniques and paradigms while continuing to push the field of HFpEF forward beyond conventional clinical and research strategies. Ultimately, although we have a long road ahead, our hope is that precision medicine will enable better, more targeted, and more efficacious treatments to ameliorate the challenging HFpEF syndrome.

\section{Acknowledgments}

Funding: Dr. Shah is supported by National Institutes of Health R01 HL107577 and R01 HL127028; and American Heart Association \#16SFRN28780016 and \#15CVGPSD27260148.

\section{References}

1. Shah SH, Arnett D, Houser SR, Ginsburg GS. Opportunities for the Cardiovascular Community in the Precision Medicine Initiative. Circulation. 2016

2. Shah SJ, Katz DH, Deo RC. Phenotypic spectrum of heart failure with preserved ejection fraction. Heart Fail Clin. 2014; 10(3):407-418. [PubMed: 24975905]

3. Bordicchia M, Liu D, Amri EZ, Ailhaud G, Dessi-Fulgheri P, Zhang C, et al. Cardiac natriuretic peptides act via p38 MAPK to induce the brown fat thermogenic program in mouse and human adipocytes. J Clin Invest. 2012; 122(3):1022-1036. [PubMed: 22307324]

4. Deo RC. Machine Learning in Medicine. Circulation. 2015; 132(20):1920-1930. [PubMed: 26572668]

5. Chirinos JA. Deep Phenotyping of Systemic Arterial Hemodynamics in HFpEF (Part 2): Clinical and Therapeutic Considerations. J Cardiovasc Transl Res. 2017

6. Chirinos JA. Deep Phenotyping of Systemic Arterial Hemodynamics in HFpEF (Part 1): Physiologic and Technical Considerations. J Cardiovasc Transl Res. 2017

7. Kao DP, Stevens LM, Hinterberg MA, Gorg C. Phenotype-Specific Association of SingleNucleotide Polymorphisms with Heart Failure and Preserved Ejection Fraction: a Genome-Wide Association Analysis of the Cardiovascular Health Study. J Cardiovasc Transl Res. 2017

8. Katz DH, Deo RC, Aguilar FG, Selvaraj S, Martinez EE, Beussink-Nelson L, et al. Phenomapping for the Identification of Hypertensive Patients with the Myocardial Substrate for Heart Failure with Preserved Ejection Fraction. J Cardiovasc Transl Res. 2017

9. Kriegel AJ, Gartz M, Afzal MZ, de Lange WJ, Ralphe JC, Strande JL. Molecular Approaches in HFpEF: MicroRNAs and iPSC-Derived Cardiomyocytes. J Cardiovasc Transl Res. 2016

10. Luo Y, Ahmad FS, Shah SJ. Tensor Factorization for Precision Medicine in Heart Failure with Preserved Ejection Fraction. J Cardiovasc Transl Res. 2017

11. Jonnalagadda SR, Adupa AK, Garg RP, Corona-Cox J, Shah SJ. Text Mining of the Electronic Health Record: An Information Extraction Approach for the Automated Identification and Subphenotyping of HFpEF Patients for Clinical Trials. J Cardiovasc Transl Res. 2017

12. Shah SJ. Clinical Trial Designs for Precision Medicine in Heart Failure with Preserved Ejection Fraction. J Cardiovasc Transl Res. 2017 
13. Redfield MM. Heart Failure with Preserved Ejection Fraction. N Engl J Med. 2016; 375(19):18681877. [PubMed: 27959663]

14. Roger VL. Epidemiology of heart failure. Circ Res. 2013; 113(6):646-659. [PubMed: 23989710]

15. Oktay AA, Rich JD, Shah SJ. The emerging epidemic of heart failure with preserved ejection fraction. Curr Heart Fail Rep. 2013; 10(4):401-410. [PubMed: 24078336]

16. Shah SJ, Heitner JF, Sweitzer NK, Anand IS, Kim HY, Harty B, et al. Baseline characteristics of patients in the treatment of preserved cardiac function heart failure with an aldosterone antagonist trial. Circ Heart Fail. 2013; 6(2):184-192. [PubMed: 23258572]

17. Owan TE, Hodge DO, Herges RM, Jacobsen SJ, Roger VL, Redfield MM. Trends in prevalence and outcome of heart failure with preserved ejection fraction. N Engl J Med. 2006; 355(3):251259. [PubMed: 16855265]

18. Sharma K, Kass DA. Heart failure with preserved ejection fraction: mechanisms, clinical features, and therapies. Circ Res. 2014; 115(1):79-96. [PubMed: 24951759]

19. Pitt B, Pfeffer MA, Assmann SF, Boineau R, Anand IS, Claggett B, et al. Spironolactone for heart failure with preserved ejection fraction. N Engl J Med. 2014; 370(15):1383-1392. [PubMed: 24716680]

20. Adamson PB, Abraham WT, Bourge RC, Costanzo MR, Hasan A, Yadav C, et al. Wireless pulmonary artery pressure monitoring guides management to reduce decompensation in heart failure with preserved ejection fraction. Circ Heart Fail. 2014; 7(6):935-944. [PubMed: 25286913]

21. Yusuf S, Pfeffer MA, Swedberg K, Granger CB, Held P, McMurray JJ, et al. Effects of candesartan in patients with chronic heart failure and preserved left-ventricular ejection fraction: the CHARMPreserved Trial. Lancet. 2003; 362(9386):777-781. [PubMed: 13678871]

22. Massie BM, Carson PE, McMurray JJ, Komajda M, McKelvie R, Zile MR, et al. Irbesartan in patients with heart failure and preserved ejection fraction. N Engl J Med. 2008; 359(23):24562467. [PubMed: 19001508]

23. Redfield MM, Chen HH, Borlaug BA, Semigran MJ, Lee KL, Lewis G, et al. Effect of phosphodiesterase-5 inhibition on exercise capacity and clinical status in heart failure with preserved ejection fraction: a randomized clinical trial. JAMA. 2013; 309(12):1268-1277. [PubMed: 23478662]

24. Redfield MM, Anstrom KJ, Levine JA, Koepp GA, Borlaug BA, Chen HH, et al. Isosorbide Mononitrate in Heart Failure with Preserved Ejection Fraction. N Engl J Med. 2015; 373(24): 2314-2324. [PubMed: 26549714]

25. Shah SJ, Kitzman DW, Borlaug BA, van Heerebeek L, Zile MR, Kass DA, et al. PhenotypeSpecific Treatment of Heart Failure With Preserved Ejection Fraction: A Multiorgan Roadmap. Circulation. 2016; 134(1):73-90. [PubMed: 27358439]

26. Kitzman DW, Shah SJ. The HFpEF Obesity Phenotype: The Elephant in the Room. J Am Coll Cardiol. 2016; 68(2):200-203. [PubMed: 27386774]

27. Borlaug BA. The pathophysiology of heart failure with preserved ejection fraction. Nat Rev Cardiol. 2014; 11(9):507-515. [PubMed: 24958077]

28. Shah SJ, Cogswell R, Ryan JJ, Sharma K. How to Develop and Implement a Specialized Heart Failure with Preserved Ejection Fraction Clinical Program. Curr Cardiol Rep. 2016; 18(12):122. [PubMed: 27796858]

29. Burkhoff D, Maurer MS, Joseph SM, Rogers JG, Birati EY, Rame JE, et al. Left atrial decompression pump for severe heart failure with preserved ejection fraction: theoretical and clinical considerations. JACC Heart Fail. 2015; 3(4):275-282. [PubMed: 25770409]

30. Shah SJ. Matchmaking for the optimization of clinical trials of heart failure with preserved ejection fraction: no laughing matter. J Am Coll Cardiol. 2013; 62(15):1339-1342. [PubMed: 23916923]

31. Shah SJ, Katz DH, Selvaraj S, Burke MA, Yancy CW, Gheorghiade M, et al. Phenomapping for novel classification of heart failure with preserved ejection fraction. Circulation. 2015; 131(3): 269-279. [PubMed: 25398313]

32. Kao DP, Lewsey JD, Anand IS, Massie BM, Zile MR, Carson PE, et al. Characterization of subgroups of heart failure patients with preserved ejection fraction with possible implications for prognosis and treatment response. Eur J Heart Fail. 2015; 17(9):925-935. [PubMed: 26250359] 
33. LeCun Y, Bengio Y, Hinton G. Deep learning. Nature. 2015; 521(7553):436-444. [PubMed: 26017442]

34. Esteva A, Kuprel B, Novoa RA, Ko J, Swetter SM, Blau HM, et al. Dermatologist-level classification of skin cancer with deep neural networks. Nature. 2017; 542(7639):115-118. [PubMed: 28117445]

35. Gulshan V, Peng L, Coram M, Stumpe MC, Wu D, Narayanaswamy A, et al. Development and Validation of a Deep Learning Algorithm for Detection of Diabetic Retinopathy in Retinal Fundus Photographs. JAMA. 2016; 316(22):2402-2410. [PubMed: 27898976]

36. Miotto R, Li L, Kidd BA, Dudley JT. Deep Patient: An Unsupervised Representation to Predict the Future of Patients from the Electronic Health Records. Sci Rep. 2016; 6:26094. [PubMed: 27185194]

37. Paulus WJ, Tschope C. A novel paradigm for heart failure with preserved ejection fraction: comorbidities drive myocardial dysfunction and remodeling through coronary microvascular endothelial inflammation. J Am Coll Cardiol. 2013; 62(4):263-271. [PubMed: 23684677]

38. Franssen C, Chen S, Unger A, Korkmaz HI, De Keulenaer GW, Tschope C, et al. Myocardial Microvascular Inflammatory Endothelial Activation in Heart Failure With Preserved Ejection Fraction. JACC Heart Fail. 2016; 4(4):312-324. [PubMed: 26682792]

39. Hall G, Rowell J, Farinelli F, Gbadegesin RA, Lavin P, Wu G, et al. Phosphodiesterase 5 inhibition ameliorates angiontensin II-induced podocyte dysmotility via the protein kinase G-mediated downregulation of TRPC6 activity. Am J Physiol Renal Physiol. 2014; 306(12):F1442-1450. [PubMed: 24740790]

40. Hidalgo C, Saripalli C, Granzier HL. Effect of exercise training on post-translational and posttranscriptional regulation of titin stiffness in striated muscle of wild type and IG KO mice. Arch Biochem Biophys. 2014; 552-553:100-107.

41. Hoke NN, Salloum FN, Kass DA, Das A, Kukreja RC. Preconditioning by phosphodiesterase-5 inhibition improves therapeutic efficacy of adipose-derived stem cells following myocardial infarction in mice. Stem Cells. 2012; 30(2):326-335. [PubMed: 22102597]

42. Yin J, Kukucka M, Hoffmann J, Sterner-Kock A, Burhenne J, Haefeli WE, et al. Sildenafil preserves lung endothelial function and prevents pulmonary vascular remodeling in a rat model of diastolic heart failure. Circ Heart Fail. 2011; 4(2):198-206. [PubMed: 21216837]

43. Anjan VY, Loftus TM, Burke MA, Akhter N, Fonarow GC, Gheorghiade M, et al. Prevalence, clinical phenotype, and outcomes associated with normal B-type natriuretic peptide levels in heart failure with preserved ejection fraction. Am J Cardiol. 2012; 110(6):870-876. [PubMed: 22681864]

44. Gupta DK, Wang TJ. Natriuretic Peptides and Cardiometabolic Health. Circ J. 2015; 79(8):16471655. [PubMed: 26103984]

45. Khan AM, Cheng S, Magnusson M, Larson MG, Newton-Cheh C, McCabe EL, et al. Cardiac natriuretic peptides, obesity, and insulin resistance: evidence from two community-based studies. J Clin Endocrinol Metab. 2011; 96(10):3242-3249. [PubMed: 21849523]

46. Cheng S, Fox CS, Larson MG, Massaro JM, McCabe EL, Khan AM, et al. Relation of visceral adiposity to circulating natriuretic peptides in ambulatory individuals. Am J Cardiol. 2011; 108(7): 979-984. [PubMed: 21813106]

47. Wang TJ. The natriuretic peptides and fat metabolism. N Engl J Med. 2012; 367(4):377-378. [PubMed: 22830469]

48. Newton-Cheh C, Larson MG, Vasan RS, Levy D, Bloch KD, Surti A, et al. Association of common variants in NPPA and NPPB with circulating natriuretic peptides and blood pressure. Nat Genet. 2009; 41(3):348-353. [PubMed: 19219041]

49. Gupta DK, de Lemos JA, Ayers CR, Berry JD, Wang TJ. Racial Differences in Natriuretic Peptide Levels: The Dallas Heart Study. JACC Heart Fail. 2015; 3(7):513-519. [PubMed: 26071618]

50. Lam CS, Cheng S, Choong K, Larson MG, Murabito JM, Newton-Cheh C, et al. Influence of sex and hormone status on circulating natriuretic peptides. J Am Coll Cardiol. 2011; 58(6):618-626. [PubMed: 21798425] 
51. Obokata M, Reddy YN, Pislaru SV, Melenovsky V, Borlaug BA. Evidence Supporting the Existence of a Distinct Obese Phenotype of Heart Failure with Preserved Ejection Fraction. Circulation. 2017

52. Anand IS, Claggett B, Liu J, Shah AM, Rector TS, Shah SJ, et al. Interaction Between Spironolactone and Natriuretic Peptides in Patients With Heart Failure and Preserved Ejection Fraction: From the TOPCAT Trial. JACC Heart Fail. 2017; 5(4):241-252. [PubMed: 28359411]

53. Shah SJ, Aistrup GL, Gupta DK, O’Toole MJ, Nahhas AF, Schuster D, et al. Ultrastructural and cellular basis for the development of abnormal myocardial mechanics during the transition from hypertension to heart failure. Am J Physiol Heart Circ Physiol. 2014; 306(1):H88-100. [PubMed: 24186100]

54. Hong T, Shaw RM. Cardiac T-Tubule Microanatomy and Function. Physiol Rev. 2017; 97(1):227252. [PubMed: 27881552]

55. Shah SJ, Wasserstrom JA. Increased arterial wave reflection magnitude: a novel form of stage B heart failure? J Am Coll Cardiol. 2012; 60(21):2178-2181. [PubMed: 23103043]

56. Riggs K, Ali H, Taegtmeyer H, Gutierrez AD. The Use of SGLT-2 Inhibitors in Type 2 Diabetes and Heart Failure. Metab Syndr Relat Disord. 2015; 13(7):292-297. [PubMed: 26125313]

57. Unger ED, Dubin RF, Deo R, Daruwalla V, Friedman JL, Medina C, et al. Association of chronic kidney disease with abnormal cardiac mechanics and adverse outcomes in patients with heart failure and preserved ejection fraction. Eur J Heart Fail. 2016; 18(1):103-112. [PubMed: 26635076]

58. Akmal M, Barndt RR, Ansari AN, Mohler JG, Massry SG. Excess PTH in CRF induces pulmonary calcification, pulmonary hypertension and right ventricular hypertrophy. Kidney Int. 1995; 47(1): 158-163. [PubMed: 7731141]

59. Polsinelli VB, Shah SJ. Advances in the pharmacotherapy of chronic heart failure with preserved ejection fraction: an ideal opportunity for precision medicine. Expert Opin Pharmacother. 2017; 18(4):399-409. [PubMed: 28129699] 


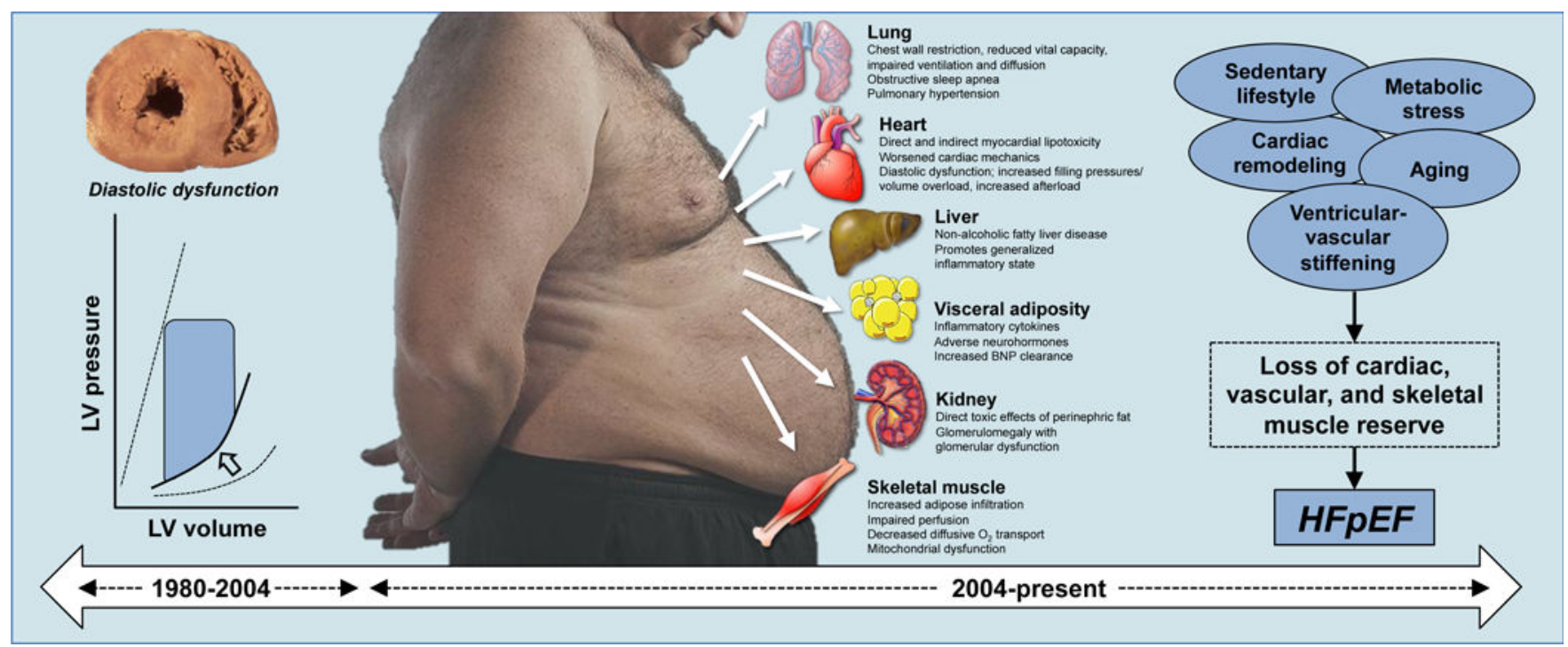

Figure 1. The Changing Paradigm of HFpEF

Prior to the early 2000s, HFpEF was generally termed "diastolic heart failure" and was thought to involve a small left ventricle with significant left ventricular hypertrophy, with diastolic dysfunction the major pathophysiologic abnormality. However, beginning in the early 2000s, it became increasingly apparent that HFpEF was more complex, with multiple pathophysiologies, and multi-organ dysfunction, with obesity playing a major role. Thus, $\mathrm{HFpEF}$ is now viewed as a multi-faceted, multi-organ disorder that involves hypertensive remodeling, ventricular-vascular stiffening, obesity/metabolic stress, aging, and sedentary lifestyle, all leading to global loss of cardiac, vascular, and peripheral reserve, which are the hallmarks of HFpEF. 


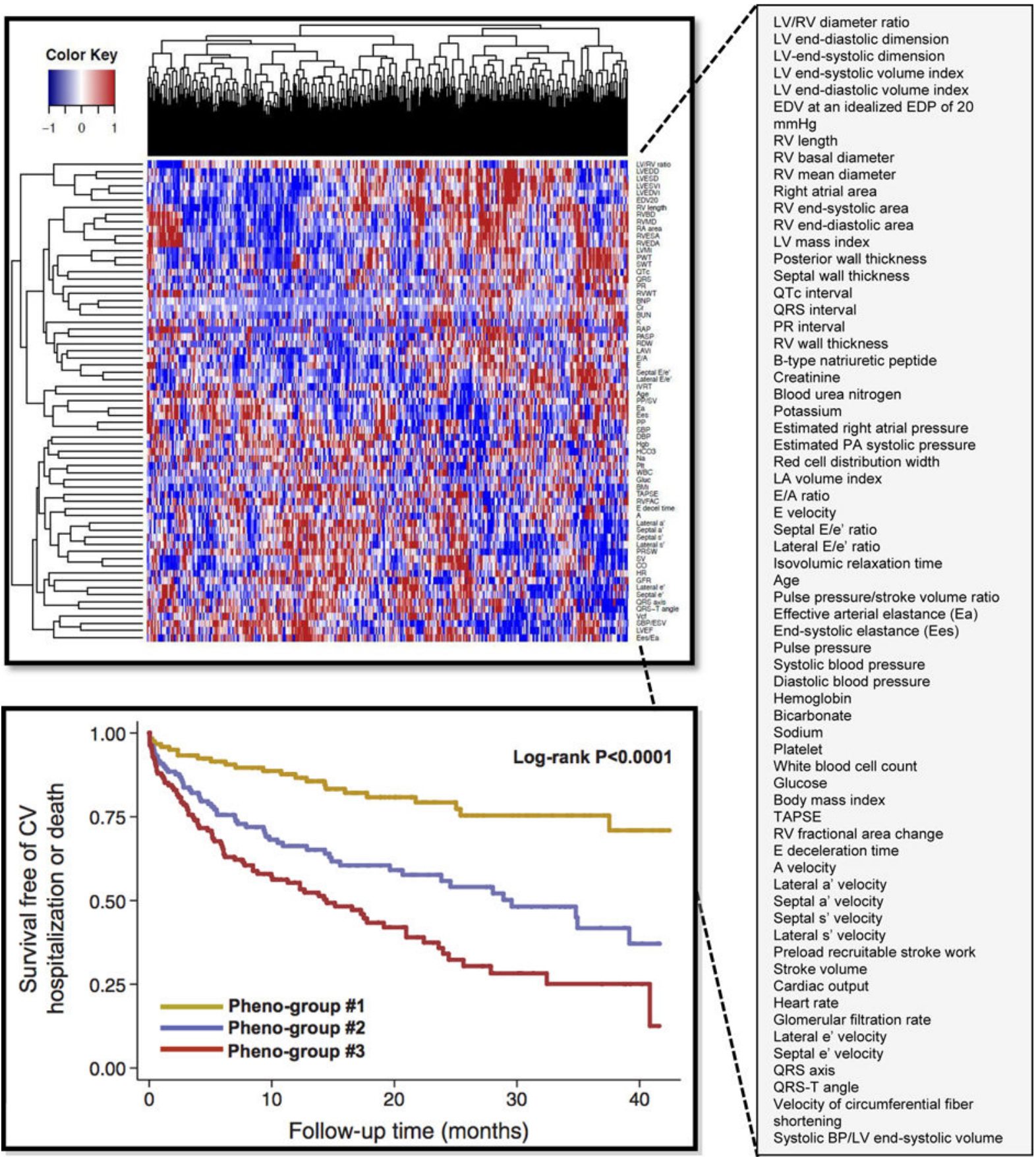

Figure 2. Phenomapping of HFpEF

Top panel: phenotype heat-map (pheno-map) of 397 patients with HFpEF. Each row represents an individual phenotype and each column represents an individual patient. Red signifies a relative increase and blue signifies a relative decrease in the phenotypic parameter, with the intensity of the color representing the magnitude of increase/decrease. The pheno-map shows that HFpEF is quite heterogeneous and that there are apparent clusters of patients that group together. Bottom panel: Kaplan-Meier curve of survival free of cardiovascular (CV) hospitalization or death. model-based clustering was ultimately used 
to stratify the HFpEF patients into 3 pheno-groups that differed significantly in outcomes. The phenotypes used in the phenomapping analysis are listed on the right of the figure. Reproduced with permission from Shah SJ, et al. Circulation 2015 (ref \#31). 

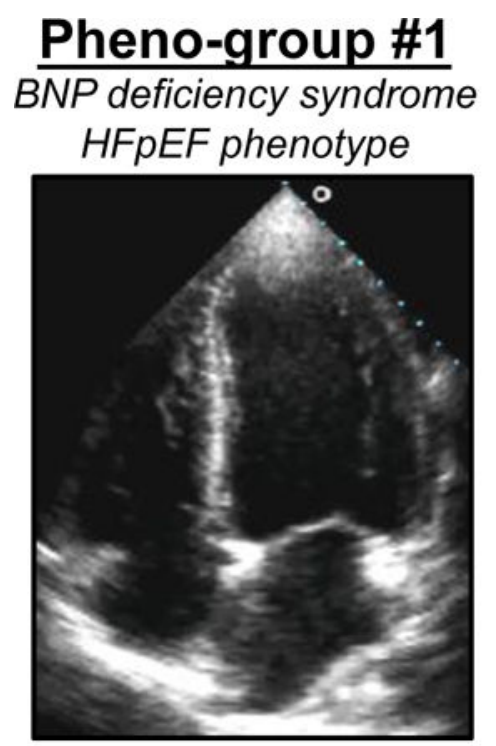

Least cardiac remodeling/ dysfunction Lowest BNP
Pheno-group \#2

Obesity-cardiometabolic HFpEF phenotype

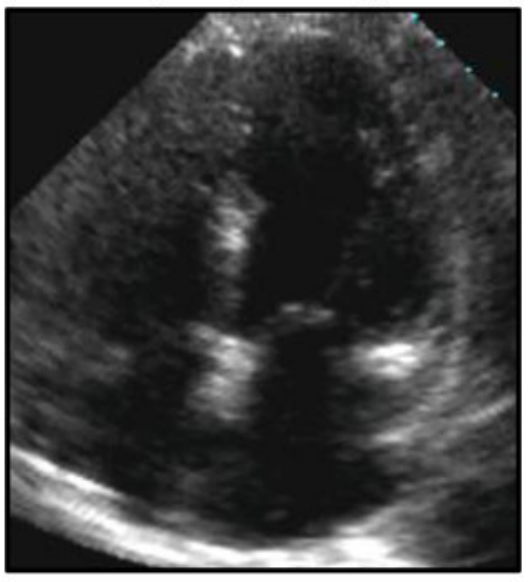

Most severely impaired myocardial relaxation Highest prevalence of
Pheno-group \#3 RV failure + cardiorenal HFpEF phenotype

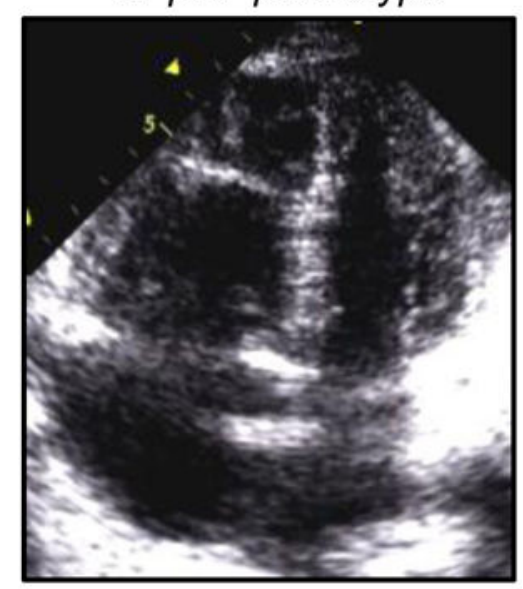

Most severe electrocardiac remodeling, RV dysfunction, renal dysfunction

Figure 3. HFpEF Pheno-Groups

Sample patients from each of the 3 HFpEF pheno-groups. The pheno-group \#1 patient has left atrial enlargement but otherwise normal-sized cardiac chambers. The pheno-group \#2 patient has biatrial enlargement and mild RV dysfunction. The pheno-group \#3 patients has biatrial enlargement and severe RV dysfunction. BNP B-type natriuretic peptide; RV = right ventricular. 

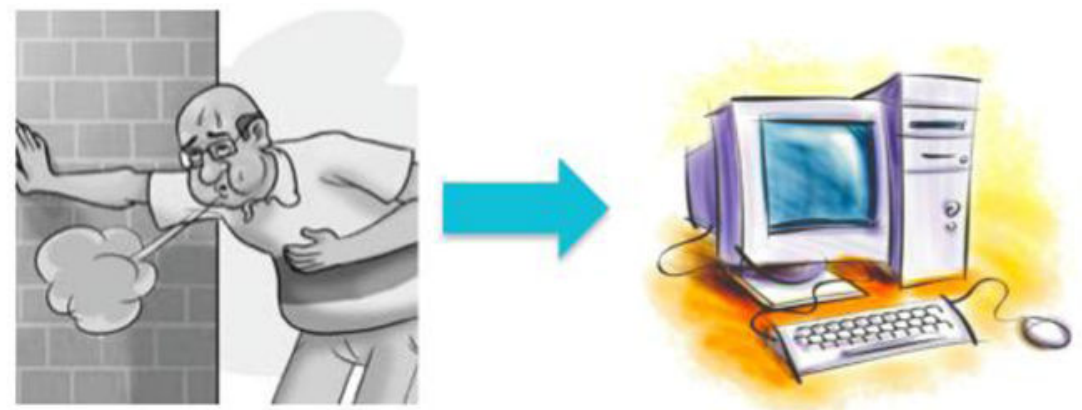

\section{$\checkmark$ History/physical}

$\checkmark$ Labs

$\checkmark$ ECG

$\checkmark$ Echo

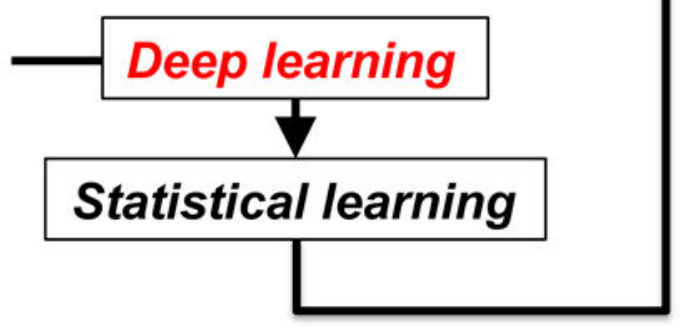

Assign

pheno-group

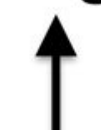

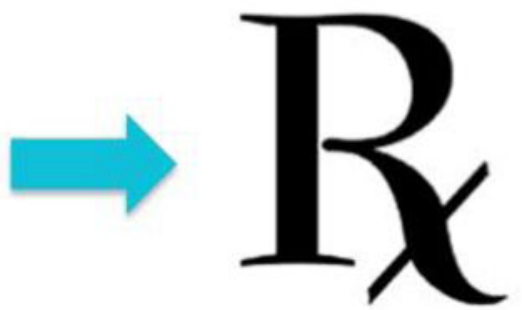

Targeted treatment, clinical trials

Figure 4. Phenomapping in the Clinic

In the future, phenomapping could be applied in the clinic, as described in the text. Patients diagnosed with HFpEF would undergo history, physical examination, laboratory testing, electrocardiography (ECG), and echocardiography. The echocardiography would be analyzed by deep learning techniques, and then all of the data would be analyzed by statistical learning techniques after which pheno-groups would be assigned. Once the patient is assigned a pheno-group, they would be directed towards targeted therapies and clinical trials. 


\begin{tabular}{|c|c|c|c|c|c|c|}
\hline & & & HFpEF Clinical & Presentation Pheno & pes & \\
\hline & & $\begin{array}{l}\text { Lung } \\
\text { Congestion }\end{array}$ & $\begin{array}{l}\text { +Chronotropic } \\
\text { Incompetence }\end{array}$ & $\begin{array}{l}\text { + Pulmonary } \\
\text { Hypertension } \\
\text { (CpcPH) }\end{array}$ & $\begin{array}{c}\text { +Skeletal } \\
\text { muscle weakness }\end{array}$ & +Atrial Fibrillation \\
\hline 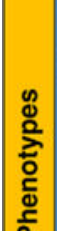 & $\begin{array}{l}\text { Overweight/obesity/ } \\
\text { metabolic syndrome/ } \\
\text { type } 2 \text { DM }\end{array}$ & $\begin{array}{l}\text { - Diuretics } \\
\text { (loop diuretic in DM) } \\
\text { - Caloric restriction } \\
\text { - Statins } \\
\text { - Inorganic nitrite/nitrate } \\
\text { - Sacubitril } \\
\text { - Spironolactone }\end{array}$ & $\begin{array}{l}\text { +Rate adaptive } \\
\text { atrial pacing }\end{array}$ & $\begin{array}{l}\text { +Pulmonary } \\
\text { vasodilators } \\
\text { (e.g. PDE5I) }\end{array}$ & $\begin{array}{l}\text { +Exercise training } \\
\text { program }\end{array}$ & $\begin{array}{l}\text { +Cardioversion } \\
\quad+\text { Rate Control } \\
\text { +Anticoagulation }\end{array}$ \\
\hline 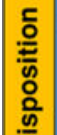 & $\begin{array}{l}+ \text { Arterial } \\
\text { hypertension }\end{array}$ & +ACEI/ARB & $\begin{array}{l}\text { +ACEI/ARB } \\
\text { + Rate adaptive } \\
\text { atrial pacing }\end{array}$ & $\begin{array}{l}\text { +ACEI/ARB } \\
\text { +Pulmonary } \\
\text { vasodilators } \\
\text { (e.g. PDE5I) }\end{array}$ & $\begin{array}{l}\text { +ACEI/ARB } \\
\text { +Exercise training } \\
\text { program }\end{array}$ & $\begin{array}{l}\text { + ACEI/ARB } \\
\text { +Cardioversion } \\
\quad \text { + Rate Control } \\
\text { +Anticoagulation }\end{array}$ \\
\hline 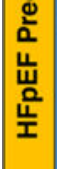 & +Renal dysfunction & $\begin{array}{l}+ \text { Ultrafiltration } \\
\text { if needed }\end{array}$ & $\begin{array}{l}\text { +Ultrafiltration } \\
\text { if needed } \\
\text { +Rate adaptive } \\
\text { atrial pacing }\end{array}$ & $\begin{array}{l}\text { +Ultrafiltration } \\
\text { if needed } \\
+ \text { Pulmonary } \\
\text { vasodilators } \\
\text { (e.g. PDE5I) }\end{array}$ & $\begin{array}{l}\text { +Ultrafiltration } \\
\text { if needed } \\
+ \text { Exercise training } \\
\text { program }\end{array}$ & $\begin{array}{l}\text { +Ultrafiltration } \\
\text { if needed } \\
\text { +Cardioversion } \\
\text { + Rate Control } \\
\text { +Anticoagulation }\end{array}$ \\
\hline & + CAD & $\begin{array}{l}\text { +ACEI } \\
\text { +Revascularization }\end{array}$ & $\begin{array}{l}\text { +ACEI } \\
\text { +Revascularization } \\
\text { +Rate adaptive } \\
\text { atrial pacing }\end{array}$ & $\begin{array}{l}\text { +ACEI } \\
\text { +Revascularization } \\
\text { +Pulmonary } \\
\text { vasodilators } \\
\text { (e.g. PDE5I) }\end{array}$ & $\begin{array}{l}\text { +ACEI } \\
\text { +Revascularization } \\
\text { +Exercise training } \\
\text { program }\end{array}$ & $\begin{array}{l}\text { +ACEI } \\
\text { + Revascularization } \\
\text { +Cardioversion } \\
\text { + Rate Control } \\
\text { + Anticoagulation }\end{array}$ \\
\hline
\end{tabular}

Figure 5. The HFpEF Treatment Matrix

The HFpEF treatment matrix involves combining HFpEF predisposition phenotypes with HFpEF clinical presentation phenotypes in order to determine the most appropriate therapy. The bolded treatment recommendations provided are based on available evidence or guidelines, whereas the other treatment recommendations are based on the expert consensus by the author group. PDE5i = phosphodiesterase type 5 inhibitor; ACEI/ARB = angiotensin receptor blocker/angiotensin converting enzyme inhibitor. Reproduced with permission from Shah SJ, et al. Circulation 2016 (ref \#25). 


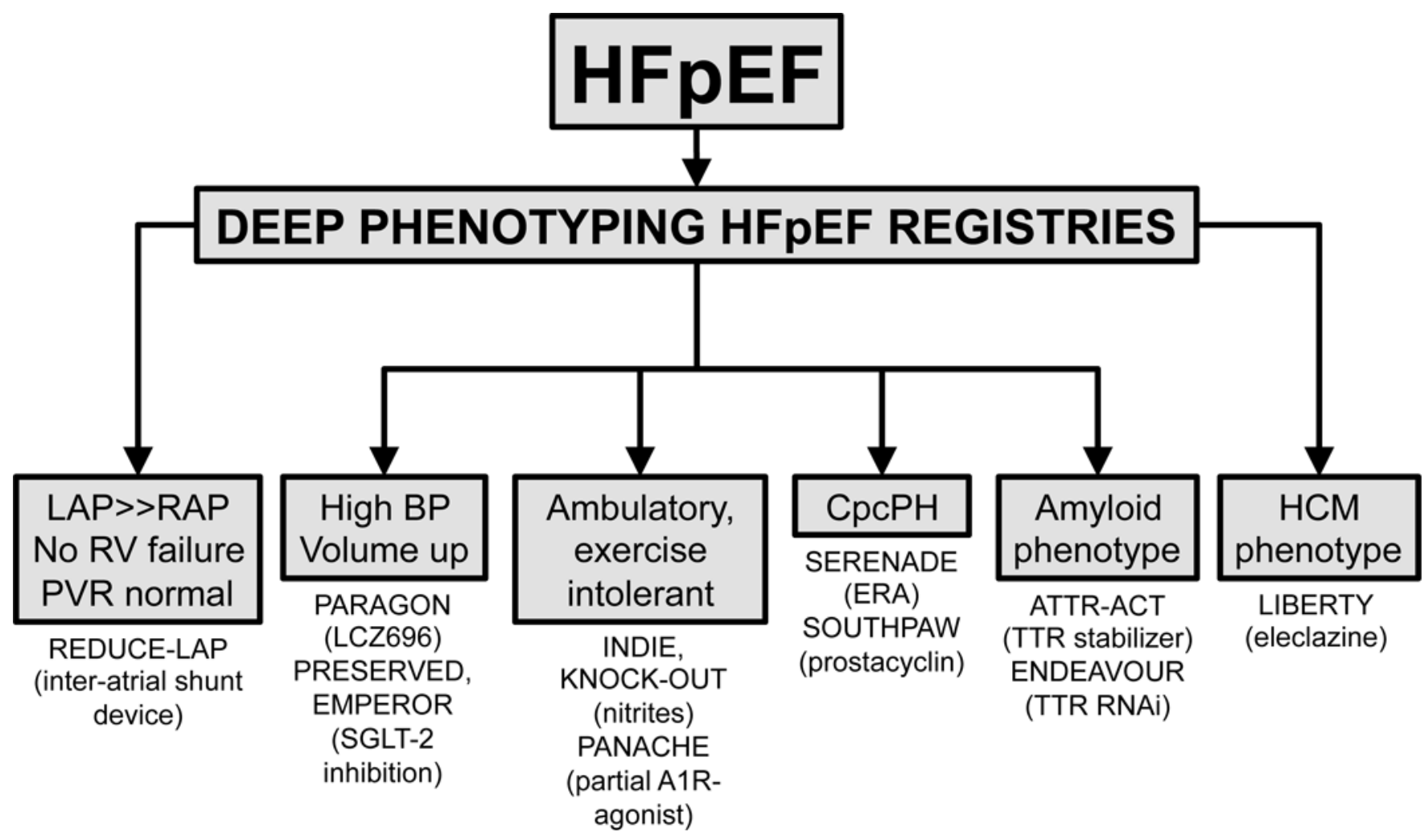

Figure 6. Precision Medicine for HFpEF

In the Northwestern University HFpEF Program, we are promoting a precision medicine approach by enrolling patients into HFpEF registries that involve deep phenotyping, after which we direct them to the most appropriate HFpEF clinical trial based on their underlying etiology or pathophysiology. LAP = left atrial pressure; RAP = right atrial pressure; $\mathrm{BP}=$ blood pressure; $\mathrm{CpcPH}=$ combined post- and pre-capillary pulmonary hypertension; HCM = hypertrophic cardiomyopathy. 
Table 1

Classification Schemes for HFpEF

\begin{tabular}{|c|c|c|}
\hline Classification scheme & Categories of $\mathrm{HFpEF}$ & Description \\
\hline \multirow[t]{8}{*}{ Clinical classification [2] } & "Garden-variety" HFpEF & $\begin{array}{l}\text { Hypertension, diabetes, obesity, and/or chronic kidney } \\
\text { disease }\end{array}$ \\
\hline & CAD-HFpEF & $\begin{array}{l}\text { Typically multi-vessel CAD with prior coronary } \\
\text { revascularization }\end{array}$ \\
\hline & Right heart failure-HFpEF & $\begin{array}{l}\text { Predominant right-sided HF with or without pulmonary } \\
\text { hypertension }\end{array}$ \\
\hline & Atrial fibrillation-predominant $\mathrm{HFpEF}$ & Atrial arrhythmias dominate the clinical presentation \\
\hline & HCM-like HFpEF & $\begin{array}{l}\text { These patients do not have genetic forms of HCM, but their } \\
\text { clinical course and echocardiographic features are typical of } \\
\text { HCM }\end{array}$ \\
\hline & High-output HFpEF & Typically due to liver disease, severe anemia \\
\hline & Valvular HFpEF & Multiple moderate valvular lesions \\
\hline & Rare causes of $\mathrm{HFpEF}$ & $\begin{array}{l}\text { e.g., infiltrative cardiomyopathies, cardiotoxicities, genetic } \\
\text { cardiomyopathies }\end{array}$ \\
\hline \multirow[t]{3}{*}{ Presentation phenotypes [30] } & Exercise-induced increase in LA pressure & $\begin{array}{l}\text { These patients typically are very breathless with exertion but } \\
\text { do not have overt signs of volume overload and typically do } \\
\text { not have a history of HF hospitalization }\end{array}$ \\
\hline & Volume overload & $\begin{array}{l}\text { Signs and symptoms of volume overload; typically have a } \\
\text { history of HF hospitalization }\end{array}$ \\
\hline & RV failure + pulmonary hypertension & $\begin{array}{l}\text { Right heart failure predominates the clinical picture; often } \\
\text { pulmonary hypertension is present and systemic blood } \\
\text { pressure is reduced }\end{array}$ \\
\hline \multirow[t]{4}{*}{ Myocardial phenotypes [29] } & Type 1: HCM & Typical genetic forms of $\mathrm{HCM}$ \\
\hline & Type 2: Infiltrative & $\begin{array}{l}\text { Cardiac amyloidosis and other forms of infiltrative or } \\
\text { restrictive cardiomyopathies }\end{array}$ \\
\hline & Type 3: Non-HTN, non-LVH & No history of hypertension and LV wall thickness $<1.2 \mathrm{~cm}$ \\
\hline & Type 4: HTN & $\begin{array}{l}\text { Typical, "garden-variety" form of HFpEF with history of } \\
\text { HTN }\end{array}$ \\
\hline \multirow[t]{6}{*}{ Latent class analysis [32] } & A: Younger males with CAD, lower LVEF & \multirow{6}{*}{$\begin{array}{l}\text { Based on latent class analysis of the I-PRESERVE AND } \\
\text { CHARM-Preserved trials. The authors used latent class } \\
\text { analysis of } 11 \text { clinical features (age, gender, BMI, atrial } \\
\text { fibrillation, CAD, diabetes, hyperlipidemia, valvular disease } \\
\text { alcohol use, eGFR, and hematocrit) to find } 6 \text { distinct groups } \\
\text { of HFpEF in I-PRESERVE and validated these findings in } \\
\text { CHARM-Preserved. }\end{array}$} \\
\hline & B: Younger females with lowest NTproBNP & \\
\hline & $\begin{array}{l}\text { C: Obesity, hyperlipidemia, diabetes mellitus, } \\
\text { anemia, and renal insufficiency }\end{array}$ & \\
\hline & D: Obese females & \\
\hline & E: Older males with CAD, lowest LVEF & \\
\hline & $\begin{array}{l}\text { F: female predominance, advanced age, lower } \\
\text { BMI, atrial fibrillation, CKD, highest } \\
\text { NTproBNP }\end{array}$ & \\
\hline \multirow[t]{3}{*}{ Phenomapping [31] } & Pheno-group \#1: BNP deficiency syndrome & \multirow{3}{*}{$\begin{array}{l}\text { Based on model-based clustering of } 67 \text { continuous variables } \\
\text { (phenotypes): physical characteristics, vital signs, ECG data } \\
\text { laboratory data, and echocardiographic parameters }\end{array}$} \\
\hline & $\begin{array}{l}\text { Pheno-group \#2: Obesity-cardiometabolic } \\
\text { phenotype }\end{array}$ & \\
\hline & $\begin{array}{l}\text { Pheno-group \#3: RV failure + cardiorenal } \\
\text { phenotype }\end{array}$ & \\
\hline
\end{tabular}

$\mathrm{CAD}=$ coronary artery disease $\mathrm{HCM}=$ hypertrophic cardiomyopathy; $\mathrm{RV}=$ right ventricular; $\mathrm{HTN}=$ hypertension $\mathrm{LVH}=$ left ventricular hypertrophy; $\mathrm{LVEF}=$ left ventricular ejection fraction; $\mathrm{BMI}=$ body mass index; $\mathrm{CKD}=$ chronic kidney disease; $\mathrm{NTproBNP}=\mathrm{N}$-terminal pro-Btype natriuretic peptide. 